\title{
Defragmentation in W-S-W Elastic Optical Networks
}

\author{
Remigiusz Rajewski \\ Faculty of Electronics and Telecommunications, Poznan University of Technology, Poznań, Poland
}

\begin{abstract}
In most cases defragmentation occurs, in elastic optical networks, in the links between the network's nodes. In this article, defragmentation in an elastic optical network's node is investigated. The W-S-W switching architecture has been used as a node. A short description of a purpose-built simulator is introduced. Several methods of defragmentation which are implemented in this simulator are described as well.
\end{abstract}

Keywords-W-S-W switching fabric, defragmentation.

\section{Introduction}

Nowadays, a typical optical WDM network offers enough sufficient bandwidth. However, it is highly probable that in the nearest future it will not be sufficient to handle a quickly increasing online traffic. Of course, a higher transmission speed could be used to solve that problem, but an optical path with the speed of $100 \mathrm{~Gb} / \mathrm{s}, 400 \mathrm{~Gb} / \mathrm{s}$ or even $1 \mathrm{~Tb} / \mathrm{s}$ is not needed by all users. Such speeds will be used mostly by network operators inside the core network. Hence, some cost-effective and scalable solutions to convey such diverse traffic will be required. Therefore, the use of Elastic Optical Networks (EONs) has been proposed [1], enabling flexible assignment of optical bandwidth. The total optical bandwidth is divided into a lot of frequency slots, where one such frequency slot constitutes the smallest amount of optical bandwidth which can be assigned to an optical path. Therefore, any connection could demand a different number of such slots. In general, one connection demands $m$ such slots. Currently, the slot width granularity equals $12.5 \mathrm{GHz}$, and it is referred to as a Frequency Slots Unit (FSU) [2].

EONs make bandwidth management easier. However, they offer also new challenges, such as, for instance, spectrum fragmentation. A sequence of connection and disconnection operations caused by the dynamic nature of the network's operation sooner or later results in the existence of non-aligned, isolated, and small-size blocks of spectrum segments. These segments can seldom be used for future connections. In most cases this results in a low spectrum utilization rate and a high probability of blocking. Therefore, the use of different defragmentation techniques allows to set up some, or sometimes all connections which nor- mally will not be set up due to improper utilization of the spectrum.

Table 1

Abbreviations used in the paper

\begin{tabular}{|c|c|}
\hline Abbreviation & Description \\
\hline BV-WCS & $\begin{array}{c}\text { Bandwidth-Variable Wavelength } \\
\text { Converting Switch }\end{array}$ \\
\hline BV-WSSS & $\begin{array}{c}\text { Bandwidth-Variable Wavelength } \\
\text { Selective Space Switch }\end{array}$ \\
\hline BV-WSS & $\begin{array}{c}\text { Bandwidth-Variable Wavelength } \\
\text { Selective Switch }\end{array}$ \\
\hline EON & Elastic Optical Network \\
\hline FSU & Frequency Slot Unit \\
\hline NED & Network Elements Description \\
\hline $\mathrm{PC}$ & Passive Coupler \\
\hline S-W-S & $\begin{array}{c}\text { Space-Wavelength-Space switching } \\
\text { fabric }\end{array}$ \\
\hline TWBC & $\begin{array}{c}\text { Tunable Waveband Bandwidth } \\
\text { Converters }\end{array}$ \\
\hline W-S-W & $\begin{array}{l}\text { Wavelength-Space-Wavelength } \\
\text { switching fabric }\end{array}$ \\
\hline
\end{tabular}

Table 2

Symbols used in the paper

\begin{tabular}{|c|c|}
\hline Symbol & Description \\
\hline \hline$c$ & Number of TWBCs \\
\hline$k$ & Number of FSUs in each interstage fiber \\
\hline$m$ & $\begin{array}{c}\text { Number of FSUs occupied by one } \\
\text { connection }\end{array}$ \\
\hline$m_{\max }$ & $\begin{array}{c}\text { Maximum number of FSUs } \\
\text { occupied by one connection }\end{array}$ \\
\hline$n$ & Number of FSUs in each input/output fiber \\
\hline$p$ & Number of switches in the center stage \\
\hline$q$ & $\begin{array}{c}\text { Number of input/output fibers in each } \\
\text { input/output switching element }\end{array}$ \\
\hline$r$ & Number of switches in the input/output stage \\
\hline
\end{tabular}


Several architectures of elastic optical switching nodes are known [3]-[5]. Recently, new architectures of EONs, referred to as Wavelength-Space-Wavelength (W-S-W) [6] and Space-Wavelength-Space (S-W-S) [7] were proposed. In this paper, two instances of the W-S-W architecture are considered, called WSW1 and WSW2, respectively [8]. Some abbreviations and symbols used in this paper have already been introduced, and some will be defined later. For the reader's convenience, they are presented in Tables 1 and 2 .

The remaining portion of the paper is organized as follows. In Section 2 a short description of the EON architectures used is provided. In Section 3 problem statement, and in Section 4 defragmentation methods are described. Section 5 introduces the simulator which allows to simulate W-S-W EONs. The last Section presents conclusions and the future work.

\section{EON's Architectures}

As mentioned before, two W-S-W switching architectures are considered in this paper: WSW1 and WSW2. In paper [9], only the WSW1 structure was implemented in the simulator proposed in Section 5. As the simulator in question still remains in the development phase, both WSW1 and WSW2 switching fabrics are already implemented.

The WSW1 switching fabric (see Fig. 1a) consists of $r$ Bandwidth-Variable Wavelength Converting Switches (BV-WCSs) with the capacity of $1 \times 1$ in the first and third stages, and of one Bandwidth-Variable Wavelength Selective Space Switch (BV-WSSS) with the capacity of $r \times r$ in the center stage. Each BV-WCS contains one BandwidthVariable Wavelength Selective Switch (BV-WSS), one Passive Coupler (PC), and $c$ Tunable Waveband Bandwidth
Converters (TWBCs). The role of BV-WSS is to direct connections from the input fiber to different TWBCs, one connection to one TWBC. In the TWBC, the connection is moved from one set of FSUs (one frequency slot) to another set of FSUs (another frequency slot). After conversion in TWBCs, all connections are combined to the output fiber by the PC. In turn, one BV-WSSS has $r$ BV-WSSs and $r$ PCs. For a detailed description of the WSW1 switching fabric see [8].

The WSW2 switching fabric (see Fig. 1b) consists of $r$ BVWCSs with the capacity of $q \times p$ in the first stage, $r$ BVWCSs with the capacity of $p \times q$ in the third stage, and $p$ BV-WSSSs with the capacity of $r \times r$ in the center stage. Each BV-WCS of the first stage contains $q$ BV-WSS, $p$ PCs and $c$ TWBCs. Each BV-WCS of the third stage contains $p$ BV-WSS, $q$ PCs and $c$ TWBCs. For WSW2, $c=q p$. The role of BV-WSS is, similarly as in the WSW1 switching fabric, to direct connections from the input fiber to different TWBCs, one connection to one TWBC. Then, in TWBC, the connection is moved from one set to another set of FSUs. After spectrum conversion, all connections are combined by PC to the output fiber. In turn, each BV-WSSS has $r$ BV-WSSs and $r$ PCs. For a detailed description of the WSW2 switching fabric see [8].

Each input and each output fiber in the W-S-W switching fabric has $n$ FSUs and each interstage fiber has $k$ FSUs (see Fig. 1). As mentioned before, a new connection could require $m$ frequency slots, where $m$ is typically limited by $1 \leq m \leq m_{\max } \leq n$. Of course, the following is always true: $n \leq k$.

\section{Defragmentation}

Defragmentation of EONs very often occurs at the network level [10]-[12]. This means that input and output node's

(a)

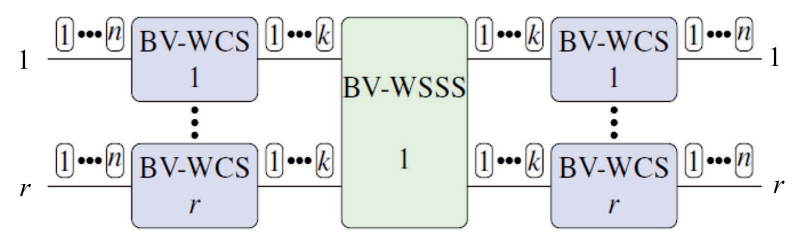

(b)

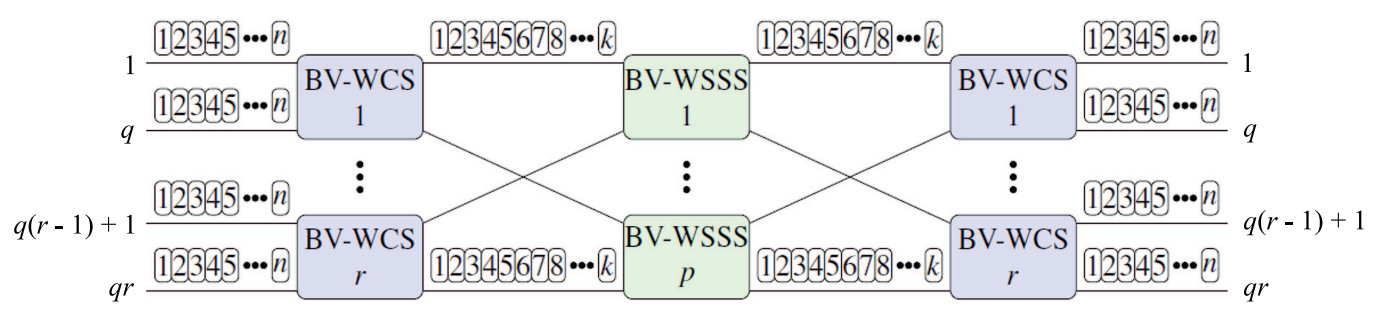

Fig. 1. W-S-W elastic optical networks: (a) WSW1 structure, (b) WSW2 structure. 
FSUs are defragmented according to the network state, and for such a defragmentation it is not important which structure of the network node is used. However, from the node point of view, defragmentation could be performed inside the node as well. Defragmentation in an EON node occurs inside interstage links only. This means that the order of FSUs at each input, as well as at each output link of the W-S-W switching fabric will not be changed. The only place where the order of FSUs can be changed is at the input and output link of the BV-WSSS switch.

The frequency slot unit defragmentation algorithm needs to address the following questions: when to defragment, what to defragment, and how to defragment?

\subsection{When to Defragment?}

Defragmentation in the WSW1 or in the WSW2 switching nodes could be performed at a different moments:

- The first moment of defragmentation is when an $m$-slot connection has just been disconnected and $m$ free FSUs have appeared in the switching node for new, future connection(s);

- The second moment of defragmentation is when a new $m$-slot connection appears in a node and there are enough FSUs to establish this connection, with those FSUs not being adjacent, however.

\subsection{What for Defragment?}

Defragmentation allows to establish a new connection when there are enough FSUs in a switching node, with the said FSUs not being adjacent to each other, however. There are several methods of defragmentation and they differ in the in which the unused FSUs are ordered. Therefore, choosing the right defragmentation method enables to reorder FSUs in the optical spectrum, which means that after the defragmentation process there will be enough adjacent FSUs to establish a new connection.

\subsection{How to Defragment?}

Several methods used to defragment FSUs may be distinguished: re-optimization [13], make-before-break [14], push-and-pull [15], and hop-tuning [16]. All of them are commonly used for defragmentation of an available spectrum used in links between EON nodes. In this article they were used to defragment an optical spectrum inside a W-S-W node.

\section{Defragmentation Methods}

In a special simulator, the four methods referred to above are used to defragment an optical bandwidth inside an EON node There are, of course, more known methods of defragmentation. However, they are have not yet been implemented in the simulator available. The simulator is described shortly in Section 5 and it is still under development.

\subsection{Re-optimization}

In the re-optimization method, all existing connections have to be disconnected and set up once again. The advantage of this method is that no additional transmitters are required. The time of defragmentation is a disadvantage. Sometimes, this lead time is very long. A simple example showing how this method workings is presented in Fig. 2. (a)
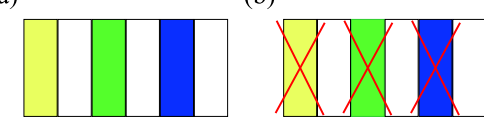

(c)

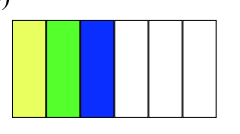

Fig. 2. Re-optimization defragmentation method: (a) step 0 state before defragmentation, (b) step 1 - disconnecting all existing connections, (c) step 2 - setting up all connections once again (state after defragmentation). (See color pictures online at www.nit.eu/publications/journal-jtit)

\subsection{Make-before-break}

In the make-before-break method, a copy of some existing connection is created in free FSUs and two identical connections exist simultaneously at a certain time. To handle this, an additional transmitter is needed. Moreover, during the defragmentation process, a new connection cannot be established due to fact that more FSUs are occupied compared to the "before defragmentation state" in the EON node. It is obvious that in order to perform defragmentation using this method, an additional number of free slots is required (in fact twice as many new FSUs are required by the new connection) and it will not always be possible to defragment an optical bandwidth inside the EON's node. A simple example showing how this method works is presented in Fig. 3 .

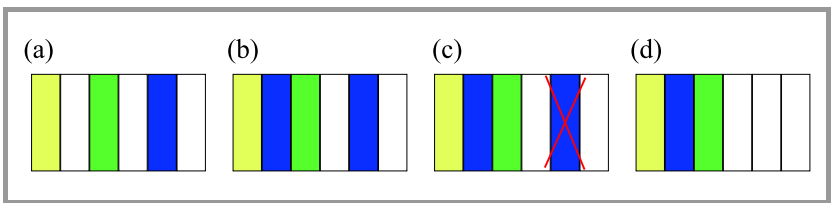

Fig. 3. Make-before-break defragmentation method: (a) step $0-$ state before defragmentation, (b) step 1 - creating a copy of the existing (blue) connection, (c) step 2 - disconnecting original connection, (d) step 3 - state after defragmentation.

\subsection{Push-and-pull}

In the push-and-pull method, some existing connections are moved within the optical bandwidth. Any connection may 
be moved inside the free adjacent FSUs, until the connection in question becomes adjacent to the other connection. In the case in which there are no free FSUs adjacent to the connection under consideration, this connection cannot be moved to other FSUs. A simple example showing how this method works is presented in Fig. 4. (a)

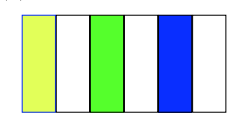

(b)

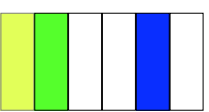

(c)

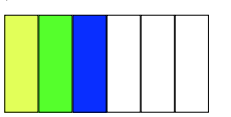

Fig. 4. Push-and-pull defragmentation method: (a) step 0 - state before defragmentation, (b) step 1 - moving the second (green) connection to the left side, (c) step 2 - moving the third (blue) connection to the left side (state after defragmentation).

\subsection{Hop-tuning}

In the hop-tuning method, an existing connection is moved to any free FSUs that are not necessarily adjacent to the connection under consideration. Unlike with the makebefore-break and push-and-pull methods, this method allows to move several connections at the same time. This is a big advantage, as the time needed for such a defragmentation is very often shorter than $1 \mu \mathrm{s}$. In this method, there is no need to use additional transmitters, which is another advantage. A simple example showing how this method works is presented in Fig. 5. (a)

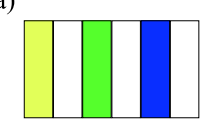

(b)

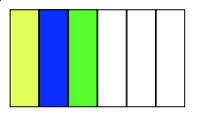

Fig. 5. Hop-tuning defragmentation method: (a) step 0 - state before defragmentation, (b) step 1 - moving (black) connection (state after defragmentation).

\section{Simulator}

In order to simulate the EON WSW1 architecture, a special software simulator has been developed, based on the OMNeT++ discrete event simulator, version 5.0 [17]. The same tool has been expanded to enable the simulation of WSW2 switching fabrics. At present, the simulator is operating based on OMNeT++ version 5.2. All functionalities (such as the behavior of all elements) and algorithms (such as defragmentation methods) have been developed with the use of $\mathrm{C}++$. All graphical representations of each element, in turn, have been prepared with the use of Network Elements Description (NED) - a special language used in the OMNeT++ environment. OMNeT++ ensures also a variety of generators which can be used as traffic generators. The traffic is very efficient in simulating all connections appearing in the elastic optical node under consideration. It also allows to randomly choose a different size of the new connection - in the case of W-S-W, it is simply the $m$ value.

The first version of this tool was used to simulate strictsense non-blocking conditions for the WSW1 switching fabric. The results obtained were compared with these achieved in paper [8]. Then, the second version of the simulator was expanded to provide for rearrangeable nonblocking conditions in the WSW1 architecture. The results obtained were compared with these presented in paper [18]. However, in both simulator versions mentioned above, graphical representation of the WSW1 switching fabric was not possible - it merely had the shape of one or several gray elements. Therefore, a third version of the simulator was developed, where the graphical representation of a WSW1 EON was possible (see Fig. 6). Four defragmentation algorithms were added to it as well. In the current version of the simulator, a new structure of W-S-W was added, namely the WSW2 switching fabric. In the future, more defragmentation algorithms will be added and more different EON structures will be supported (like, for instance, S-W-S-type switching fabrics).

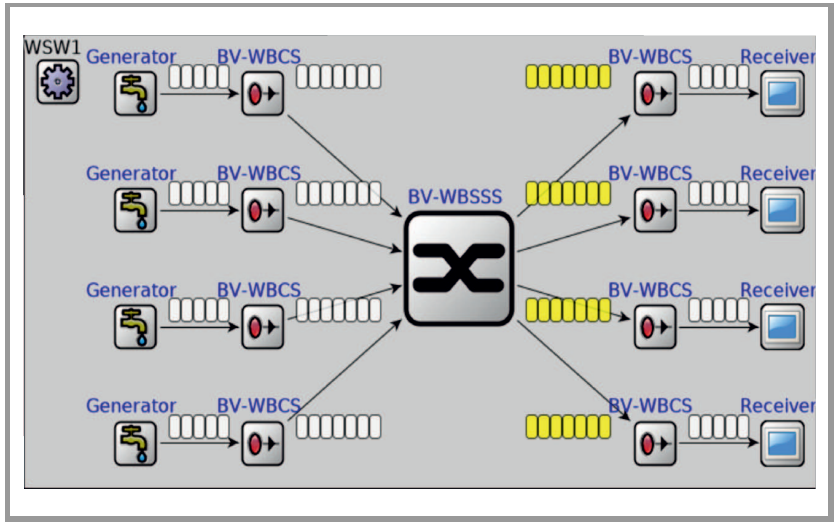

Fig. 6. WSW 1 node with $n=5, r=4$, and $k=7$ operating in the OMNeT++ environment.

The current software version uses $q r+1$ cMersenneTwister random number generators that are already available in the $\mathrm{OMNeT}++$ simulation environment. There are $q r$ different generators (one per input) which draw the number of the output to which the new connection is directed. The size of the new connection $m$, in turn, is drawn by another generator, assigning an integer from the range of 1 and $m_{\max }$. In the future, a generator responsible for the Poisson type of traffic will be added as well.

The WSW1 switching node simulator asks the user, immediately after its start-up, which parameters (like $n, r$, and $k$ ) should be used during the simulation. By specifying different values of $n, r$ and $k$, different structures of WSW1 will be obtained. For example, when $n=5, r=4$ and $k=7$, the WSW1 EON looks as presented in Fig. 6. Meanwhile, with $n=10, r=2$ and $k=15$, the WSW1 structure looks as shown in Fig. 7. 


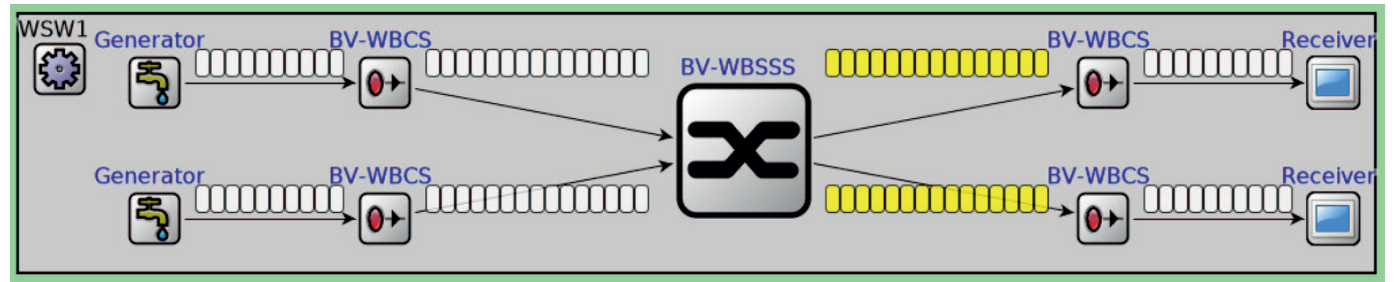

Fig. 7. WSW1 node with $n=10, r=2$, and $k=15$ operating in the OMNeT++ environment.

In the simulator, each BV-WBCS, as well as BV-WBSSS, are represented by a proper module which consists of smaller pieces, such as PCs, BV-WSSs and TWBCs. For example, for $n=5$, each BV-WBCS looks as shown in Fig. 8.

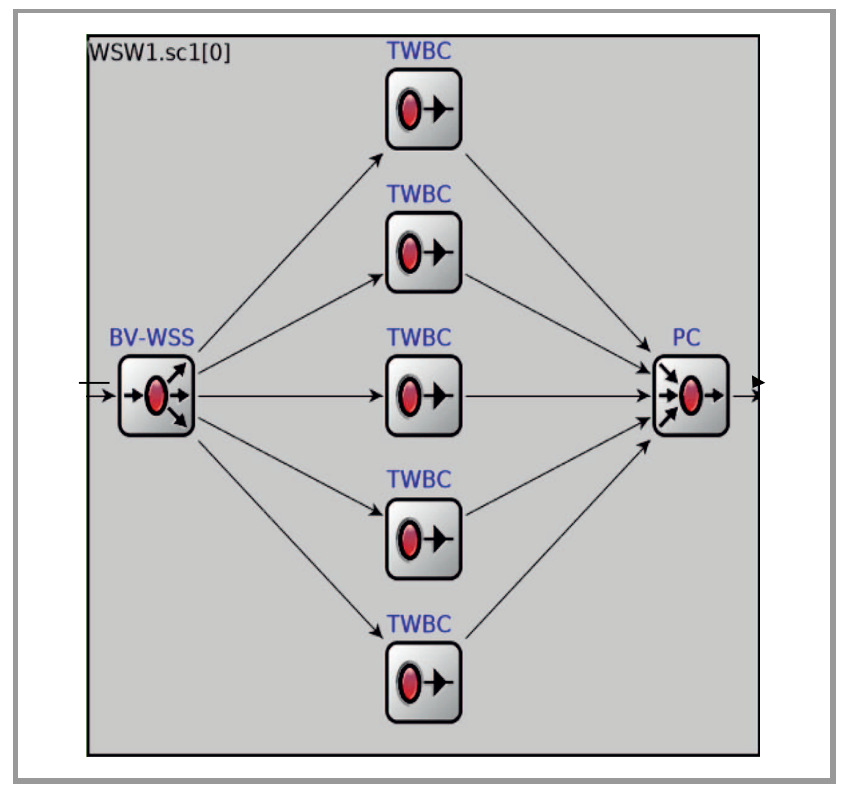

Fig. 8. The BV-WBCS switch implemented in OMNeT++.

For $r=2$, the BV-WBSSS switch looks as presented in Fig. 9, and for $r=7$, as shown in Fig. 10.

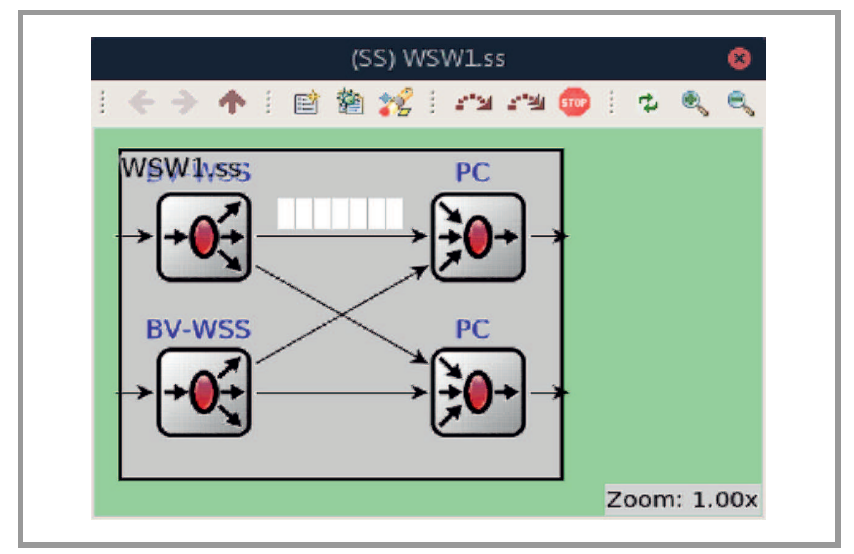

Fig. 9. The BV-WSSS switch for $r=2$.

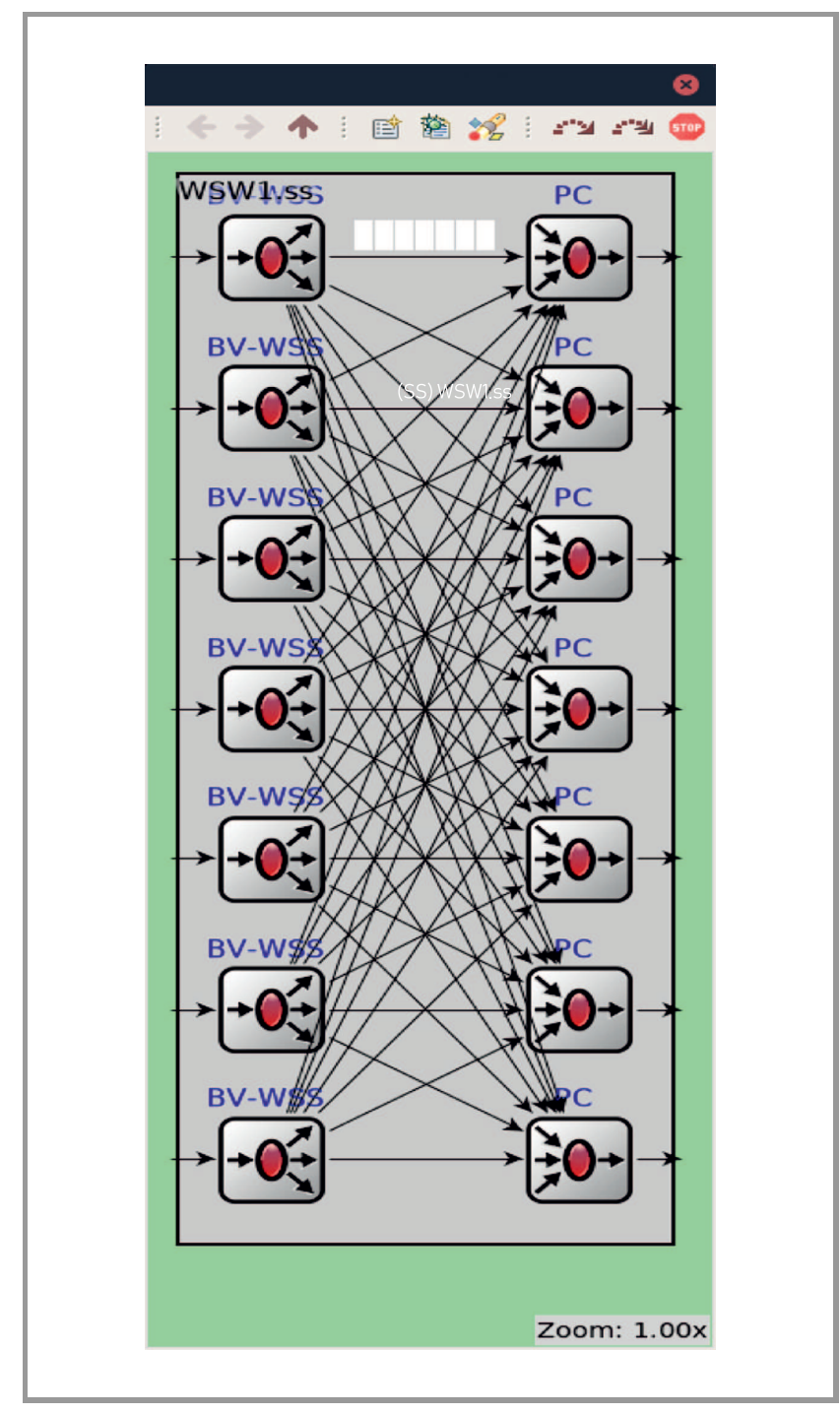

Fig. 10. The BV-WSSS switch for $r=7$.

\section{Conclusions}

Four defragmentation methods for the W-S-W architecture have been described in this paper. All of these methods were already implemented in a purpose-developed simulator. The current version of the simulator offers a graphical interface as well. However, it is still under development. The future step is to represent the S-W-S switching fabric 
(another example of an elastic optical network) in the simulator as well. Both SWS1 and SWS2 architectures will be implemented. Such a future solution will make it possible to compare both types of structures with one another. Other defragmentation methods will be deployed as well.

\section{Acknowledgments}

The work described in this paper was financed with the funds of the Ministry of Science and Higher Education for 2017, under grant 08/82/DSMK/8222.

\section{References}

[1] M. Jinno et al., "Spectrum-efficient and scalable elastic optical path network: Architecture, benefits, and enabling technologies", IEEE Communications Magazine, vol. 47, no. 11, pp. 66-73, 2009.

[2] ITU-T Recommendation G.694.1. Spectral Grids for WDM Applications: DWDM Frequency Grid, 2012.

[3] P. Zhang et al., "Comparison of node architectures for elastic optical networks with waveband conversion", China Commun., vol. 10, no. 8, pp. 77-87, 2013.

[4] Y. Chen et al., "Demonstration of petabit scalable optical switching with subband-accessibility for elastic optical networks", in Proc. The OptoElectr. and Commun. Conf. and Austr. Conf. on Opt. Fibre Technol. OECC/ACOFT, Melbourne, VIC, Australia, July 2014, pp. 350-351.

[5] B. C. Chatterjee, N. Sarma, and E. Oki, "Routing and spectrum allocation in elastic optical networks: A tutorial", IEEE Commun. Surveys and Tutor., vol. 17, no. 3, pp. 1776-1800, 2015.

[6] W. Kabaciński, M. Michalski, and M. Abdulsahib, "The strict-sense nonblocking elastic optical switch", in Proc. IEEE 16th Int. Conf. on High Perfor. Switching and Routing HPSR, Budapest, Hungary, July 2015 (doi: 10.1109/HPSR.2015.7483108).

[7] G. Danilewicz, W. Kabaciński, and R. Rajewski, "Strict-sense nonblocking space-wavelength-space switching fabrics for elastic optical network nodes", J. of Optic. Commun. and Network., vol. 8, no. 10, pp. 745-756, 2016.
[8] W. Kabaciński, M. Michalski, and R. Rajewski, "Strict-sense nonblocking W-S-W node architectures for elastic optical networks", $J$. of Lightwave Technol., vol. 34, no. 13, pp. 3155-3162, 2016.

[9] R. Rajewski, "Defragmentation in the W-S-W Elastic Optical Network," in Proc. IEICE Infor. and Commun. Technol. Forum ICTF 2017, Poznań, Poland, 2017.

[10] M. Zhang, W. Shi, L. Gong, W. Lu, and Z. Zhu, "Bandwidth defragmentation in dynamic elastic optical networks with minimum traffic disruptions", in Proc. IEEE Int. Conf. on Commun. ICC, Budapest, Hungary, 2013, pp. 3894-3898.

[11] C. You, M. Zhang, and Z. Zhu, "Reduce Spectrum Defragmentation Latency in EONs with Effective Parallelization of Connection Reconfigurations", in Proc. Opt. Fiber Commun. Conf. and Exh. OFC, San Francisco, CA, USA, 2014, pp. 1-3.

[12] S. Ba, B. C. Chatterjee, and E. Oki, "Defragmentation scheme based on exchanging primary and backup paths in $1+1$ path protected elastic optical networks", IEEE/ACM Transact. on Network., vol. 25, no. 3, pp. 1717-1731, 2017.

[13] I. Tomkos, S. Azodolmolky, J. Solé-Pareta, D. Careglio, and E. Palkopoulou, "A tutorial on the flexible optical networking paradigm: State of the art, trends, and research challenges", in Proceedings of the IEEE, vol. 102, no. 9, pp. 1317-1337, 2014.

[14] A. Pagès, J. Perelló, and S. Spadaro, "Lightpath fragmentation for efficient spectrum utilization in dynamic elastic optical networks", in Proc. 2012 16th Int. Conf. on Opt. Network Design and Modelling ONDM, Colchester, United Kingdom, 2012, pp. 1-6.

[15] F. Cugini et al., "Push-pull defragmentation without traffic disruption in flexible grid optical networks", J. of Lightwave Technol., vol. 31, no. 1, pp. 125-133, 2013.

[16] M. Zhang, Y. Yin, R. Proietti, Z. Zhu, and S. J. B. Yoo, "Spectrum defragmentation algorithms for elastic optical networks using hitless spectrum retuning techniques", Proc. 2013 Opt. Fiber Commun. Conf. and Exp. and the Nation. Fiber Optic Engineers Conf. (OFC/NFOEC), Anaheim, CA, USA, 2013, pp. 1-3.

[17] The OMNeT++ official website, 20 Oct 2017 [Online]. Available: https://omnetpp.org

[18] W. Kabaciński, R. Rajewski, and A. Al-Tameemi, "Simultaneous connections routing in elastic optical switches with limited number of connection rates", in Proc. 21st Int. Conf. on Opt. Net. Design and Models ONDM, Budapest, Hungary, 2017.

Remigiusz Rajewski - for biography, see this issue, p. 17. 